\title{
Curious Mechanism of the Dissociative Chemisorption of Ammonia on $\mathrm{Ru}(0001)$
}

\author{
Nick Gerrits*(-) and Geert-Jan Kroes*(i) \\ Leiden Institute of Chemistry, Gorlaeus Laboratories, Leiden University, P.O. Box 9502, 2300 RA Leiden, South Holland, The \\ Netherlands
}

\section{Supporting Information}

\begin{abstract}
Dissociative chemisorption of polyatomic molecules on metals, which is relevant to heterogeneous catalysis, usually proceeds through a rotationally adiabatic or rotational sudden mechanism. The reaction is usually either direct or proceeds through a trapped molecular chemisorbed state. Here, ab initio molecular dynamics is used to model the dissociative chemisorption of ammonia on $\mathrm{Ru}(0001)$. The reaction mechanism is neither rotationally adiabatic nor rotational sudden, with clearly distinct and nonstatistical initial and time-of-reaction orientation distributions. A reasonably good agreement is obtained between the computed and previously measured sticking probabilities. Under the conditions investigated, the reaction of $\mathrm{NH}_{3}$ goes through a molecular chemisorption-like state, but the reaction is direct.
\end{abstract}

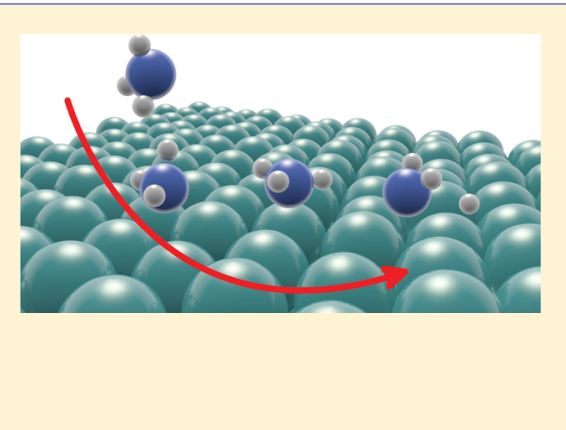

\section{INTRODUCTION}

Recent ab initio molecular dynamics (AIMD) $)^{1-7}$ and quasiclassical trajectory $(\mathrm{QCT})^{8,9}$ calculations on high-dimensional potential energy surfaces (PESs) are providing a wealth of information on the dynamics of polyatomic molecules reacting on metal surfaces. Of these, direct reactions on metal surfaces are typically either rotationally adiabatic, ${ }^{10}$ for example, water $+\mathrm{Ni}(111),{ }^{4,11}$ or in a sudden regime, ${ }^{12,13}$ for example, $\mathrm{CHD}_{3}+$ $\mathrm{Pt}(111)^{5}$ and methanol $+\mathrm{Cu}(111)^{7}$ (Figure 1a). In the former case, the initial orientation distribution of the reacting molecules is statistical [i.e., it resembles a $\sin (\theta)$ distribution of the $\theta_{\mathrm{d}}$ angle shown in Figure 1a] and is steered toward transition state (TS) values. ${ }^{4}$ In the latter case, the initial orientation distribution is already close to the orientation at the barrier geometry. ${ }^{5,7,14,15}$ Approximate methods such as the reaction path Hamiltonian approach ${ }^{16}$ often use either a rotationally adiabatic or a sudden approximation. ${ }^{13,14}$ Furthermore, if a reaction proceeds through a molecular chemisorption-like state, it is usually trapping mediated. ${ }^{3}$ However, as we will show here, ammonia reacts on $\mathrm{Ru}(0001)$ through a very different mechanism, in which both the incident orientation distribution and the distribution at the TS are nonstatistical but clearly distinct. Furthermore, the reaction is observed to be direct, even though the molecule proceeds through a molecular chemisorption-like geometry as observed in the trapping-mediated reaction.

Because of the high pressures and temperatures involved in the Haber-Bosch process, ${ }^{18}$ whereby $\mathrm{N}_{2}$ is converted to $\mathrm{NH}_{3}$, ammonia is not only a product in this process, but also a reactant, and $\mathrm{Ru}$ is a good catalyst for ammonia production. ${ }^{19}$ Therefore, predicting and understanding the reaction of ammonia on $\mathrm{Ru}(0001)$ is not only of interest for fundamental reasons, but it is also of practical importance, as $\mathrm{NH}_{3}$ is a raw material for the production of the synthetic fertilizer that helps feed a substantial part of the world's population. ${ }^{20}$ Additionally, $\mathrm{Ru}$ is the best single metal catalyst for ammonia decomposition, ${ }^{21-23}$ which is relevant to the production of $\mathrm{CO}_{x}$-free $\mathrm{H}_{2}$ for hydrogen fuel cell applications. ${ }^{22}$ In the kinetics of ammonia decomposition, the breaking of the first $\mathrm{NH}$-bond is an important step. ${ }^{22-24}$

Molecular beam sticking experiments on dissociative chemisorption of ammonia on $\mathrm{Ru}(0001)$ have been performed by Mortensen et al. ${ }^{25}$ at surface temperatures $\left(T_{\mathrm{s}}\right)$ of 475 and $1100 \mathrm{~K}$. They found the dissociation to be activated and independent of $T_{\mathrm{s}}$ at incidence energies larger than $85 \mathrm{~kJ} / \mathrm{mol}$. Consequently, they proposed a direct reaction mechanism for these conditions. For the lower $E_{\mathrm{i}}$, their detailed experiments allowed them to propose a mechanism involving a molecularly chemisorbed state reacting at defect sites. In the latter mechanism, diffusion of reactants to and products away from the defects limits the sticking at very low $T_{s}$, and desorption of $\mathrm{NH}_{3}$ prior to reaching the defects limits the trapping-mediated reaction at high $T_{s}$.

So far, only Hu et al. ${ }^{26}$ used dynamics calculations to model the experimentally measured sticking probabilities, performing QCT calculations on a twelve-dimensional (12D) PES fitted with a neural network approach. ${ }^{27}$ Their work focused on vibrational enhancement of the reaction, and they found vibrational efficacies near unity for each of the four vibrational modes of $\mathrm{NH}_{3}$. Although the dynamical behavior of the ammonia molecule was included, the metal surface atoms were kept frozen. They did not model energy transfer to the surface, even though this can play a major role in the computed reactivity for molecule-metal surface reactions. ${ }^{8,12,13}$ The Perdew, Burke, and Ernzerhof (PBE) exchange-correlation

Received: September 26, 2019

Published: October 29, 2019 


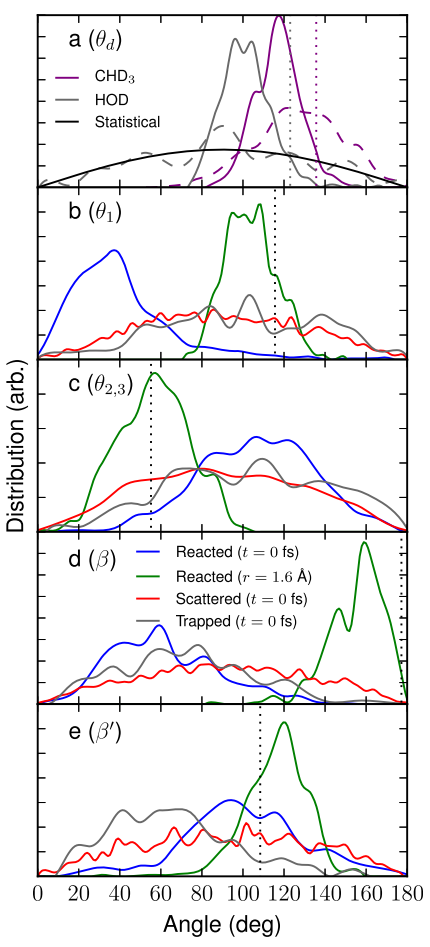

Figure 1. (a) $\theta_{\mathrm{d}}$ angle, that is, the dissociating bond, of $\mathrm{CHD}_{3}{ }^{17}$ (purple) and $\mathrm{HOD}^{4}$ (gray) for all the reacted trajectories at the initial time step (dashed lines) and when a dissociating bond reaches the TS value (solid lines). The solid black line indicates a statistical $\sin (\theta)$ distribution. The dotted lines indicate the TS values. (b-e) $\theta_{1}, \theta_{2,3}, \beta$, and $\beta^{\prime}$ angles of ammonia during the AIMD for all the reacted (blue), scattered (red), and trapped (gray) trajectories at the initial time step and when a dissociating bond reaches the TS value (green). The angles of the nondissociating hydrogen atoms with respect to the surface normal are indicated by $\theta_{2,3}$. $\beta^{\prime}$ indicates the angle between the surface normal and the umbrella axis, which is defined as the vector going from the geometric center of the three hydrogen atoms to the nitrogen atom. The dotted lines indicate the TS values belonging to the top $2 \mathrm{fcc}$ barrier geometry.

(xc) functiona ${ }^{28}$ was used. For high incidence energies the computed sticking probabilities for $\mathrm{NH}_{3}$ in its initial vibrational ground state were considerably higher (by a factor 2-2.5) than the experimental sticking probabilities, which the authors attributed to the use of the PBE functional. Indeed, this functional, like its very similar ${ }^{28}$ predecessor PW91, ${ }^{29}$ typically overestimates the reactivity of molecules on metal surfaces. ${ }^{5,30-33}$

Here, we study the reaction at the higher incidence energy conditions for which the experimentalists did not yet characterize the reaction mechanism in detail, and for which AIMD calculations can be used; for high incidence energies the system can be kept small as defects do not play an important role, and propagation times can be kept short. In the AIMD calculations, we model the motions of $\mathrm{NH}_{3}$ as well as that of the surface atoms of $\mathrm{Ru}(0001)$ explicitly. We address different aspects of the reaction mechanism, that is, the orientation distribution of the reacting molecules, and the role of the molecular chemisorption state in the reaction at high incidence energies. A density functional is used containing revised PBE $(\mathrm{RPBE})^{34}$ exchange (more repulsive than PBE exchange) and the van der Waals correlation functional of Dion et al. (vdW$\mathrm{DF} 1),{ }^{35}$ which we refer to as the RPBE-vdW-DF1 functional. We show that the reaction proceeds through an unusual mechanism, in which the initial orientation distribution of the reacting molecules is nonstatistical, but is clearly distinct from the nonstatistical distribution at the time of reaction, which resembles the orientation at the TS. Additionally, although the reaction is direct, the reacting molecules go through a geometry that is similar to the geometry that would be taken on by the molecular precursor state dominating the reaction mechanism at low $E_{\mathrm{i}}$. Compared to the earlier dynamics calculations $^{26}$ the agreement with the experimental sticking probabilities is improved.

\section{METHOD}

For the AIMD and electronic structure (density functional theory) calculations the Vienna Ab initio Simulation Package (VASP version 5.3.5) $)^{36-40}$ is used. The first Brillouin zone is sampled by a $\Gamma$-centered $4 \times 4 \times 1 k$-point grid and the plane wave basis set kinetic energy cutoff is $400 \mathrm{eV}$. Moreover, the core electrons have been represented with the projector augmented wave method. ${ }^{40,41}$ The surface is modeled using a 4 layer $(3 \times 3)$ supercell, where the top three layers have been relaxed in the $Z$ direction and a vacuum distance of $15 \AA$ is used between the slabs. Because of the use of the vdW-DF1 correlation functional, the employed vacuum distance causes a small interaction energy between the surface and the molecule in the gas phase, which effectively raises the barrier height by $3.0 \mathrm{~kJ} / \mathrm{mol}$. However, because of the computational cost, a larger vacuum distance is untractable in the AIMD. Therefore, $3.0 \mathrm{~kJ} / \mathrm{mol}$ is added to the translational energy to counteract this shift, as done and justified previously. ${ }^{17}$ In order to speed up the convergence, first order Methfessel-Paxton smearing ${ }^{42}$ with a width parameter of $0.2 \mathrm{eV}$ has been applied. The employed computational setup is confirmed to be converged within chemical accuracy ( $1 \mathrm{kcal} / \mathrm{mol}$, or $4.2 \mathrm{~kJ} / \mathrm{mol}$ ), as shown in the Supporting Information (Figure S1 and Tables S1 and S2).

TSs are obtained with the dimer method m $^{43-46}$ as implemented in the VASP Transition State Tools package (VTST) and are confirmed to be first order saddle points. Forces on the degrees of freedom are converged within 5 $\mathrm{meV} / \AA$, where only ammonia is relaxed in all its degrees of freedom, that is, when computing TSs the surface is kept fixed in its relaxed surface-vacuum geometry.

We used the RPBE-vdW functional, which is defined as

$$
E_{\mathrm{xc}}=E_{\mathrm{x}}^{\mathrm{RPBE}}+E_{\mathrm{c}}^{\mathrm{vdW}-\mathrm{DF} 1}
$$

where $E_{\mathrm{x}}^{\mathrm{RPBE}}$ is the exchange part of the $\mathrm{RPBE}^{34}$ exchangecorrelation functional and $E_{\mathrm{c}}^{\mathrm{vdW}-\mathrm{DF} 1}$ is the nonlocal van der Waals correlation functional of Dion and co-workers (vdWDF 1$).{ }^{35}$

A surface temperature of 475 and $1100 \mathrm{~K}$ is simulated in the AIMD calculations, where the atoms in the top three layers are allowed to move. The expansion of the bulk due to the surface temperature is simulated by multiplying ${ }^{48}$ the computed ideal lattice constants $(a=2.7524, c=4.3334)$ with the thermal expansion coefficients ${ }^{47}$ that are provided in Table 1 .

Because ammonia has a similar mass as methane, the parameters used to simulate the molecular beam bundles (the stream velocity and width parameters, see Table S4) are taken from ref 49, which reported experiments performed for $\mathrm{CHD}_{3}$ $+\operatorname{Pt}(111)$. For every AIMD data point at a surface temperature of 475 and $1100 \mathrm{~K}, 1000$ and 500 trajectories were run, respectively, using a time step of 0.4 fs. Other 
Table 1. Thermal Expansion Coefficients for the $a$ and $c$ Lattice Vectors $^{a}$

$\begin{array}{ccc}\text { temperature }(\mathrm{K}) & \alpha_{a}\left(\mathrm{~K}^{-1}\right) & \alpha_{c}\left(\mathrm{~K}^{-1}\right) \\ 475 & 1.00110 & 1.00168 \\ 1100 & 1.00684 & 1.01045\end{array}$

${ }^{a}$ Coefficients are taken from ref 47.

technical details of the AIMD calculations and the sampling of the initial conditions can be found in recent work ${ }^{15,17,32,49}$ and in the Supporting Information. Note that because $\mathrm{NH}_{3}$ is a symmetric top rotor, the rotational states have been described in the same manner as for $\mathrm{CHD}_{3}$, which is also a symmetric top rotor.

\section{RESULTS}

3.1. Activation Barriers and Adsorption Energies. The barrier heights and geometries obtained with the RPBE-vdW$\mathrm{DF} 1$, SRP32-vdW, ${ }^{19}$ and $\mathrm{PBE}^{28}$ functionals are shown in Tables 2 and 3 . Figure $2 \mathrm{a}-\mathrm{c}$ shows the top $2 \mathrm{fcc}$ barrier geometry obtained with the RPBE-vdW-DF1 functional and depicts the angles that are used in Table 2 . The $\theta_{1}$ angle is the angle between the dissociating bond and the surface normal. The $\beta$ angle is the angle between the surface normal and the principal axis of the $\mathrm{NH}_{2}$ fragment, which is defined as the vector going from the geometric center of the two nondissociating hydrogen atoms to the nitrogen atom. The $\gamma$ angle indicates the angle between the axis defined and the dissociating bond (see Figure $2 \mathrm{a}$ ). $Z_{\mathrm{N}}$ indicates the distance of the nitrogen atom to the surface and the length of the dissociating $\mathrm{NH}$ bond is indicated by $r$ (see Figure $2 \mathrm{~b}$ ).

Two barriers have been obtained, the top $2 \mathrm{fcc}$ and top $2 \mathrm{hcp}$ barriers, of which the top2hcp barrier height $(63.2 \mathrm{~kJ} / \mathrm{mol})$ is $2.7 \mathrm{~kJ} / \mathrm{mol}$ lower than the top $2 \mathrm{fcc}$ barrier height $(65.9 \mathrm{~kJ} /$ $\mathrm{mol})$. Moreover, in terms of the five coordinates shown in Figure $2 \mathrm{a}, \mathrm{b}$ the two barrier geometries are very similar, with an important difference being the location of the dissociating hydrogen, that is, towards the fcc and hcp hollow sites. The top $2 \mathrm{fcc}$ barrier geometry obtained by $\mathrm{Hu}$ et al. ${ }^{26}$ using the $\mathrm{PBE}$ functional is similar to the one obtained with the RPBE-vdWDF1 functional but their top $2 \mathrm{fcc}$ barrier height is $20.3 \mathrm{~kJ} / \mathrm{mol}$ lower. Moreover, with our computational setup, but with the PBE functional, we obtained a similar top2fcc barrier height and geometry as $\mathrm{Hu}$ et al., where the difference in the barrier heights is only $2.3 \mathrm{~kJ} / \mathrm{mol}$. Interestingly, for PBE the top $2 \mathrm{hcp}$ barrier height we obtained is also lower than the top $2 \mathrm{fcc}$ barrier height (by $3.1 \mathrm{~kJ} / \mathrm{mol}$ ), but the top $2 \mathrm{hcp}$ barrier was not mentioned previously by $\mathrm{Hu}$ et $\mathrm{al}^{26} \mathrm{We}$ also note that the converged surface lattice constant for PBE in this work is slightly smaller $(a=2.7148 \AA)$ than the one obtained by $\mathrm{Hu}$ et al. $^{26}(a=2.7251 \AA)$, for which the reason is unknown.

With the SRP32-vdW functional previously developed for $\mathrm{CHD}_{3}+\mathrm{Ni}(111),{ }^{17}$ barrier heights for $\mathrm{NH}_{3}+\mathrm{Ru}(0001)$ are obtained that are similar to the PBE barrier heights, although now the top $2 \mathrm{fcc}$ barrier height is $0.6 \mathrm{~kJ} / \mathrm{mol}$ lower than the top2hcp barrier height. Again, the geometries are similar to the geometries obtained with the RPBE-vdW-DF1 functional. This was also observed for the barriers of $\mathrm{CHD}_{3}$ on $\mathrm{Pt}(111)$ obtained with the PBE and SRP32-vdW functionals. ${ }^{5}$ In general, it seems that the vdW interactions mostly lower the barrier height, and do not affect the barrier geometry much: mixing in repulsive RPBE exchange (by going from PBE to SRP32 or RPBE exchange) while retaining PBE correlation would raise the barrier, but replacing $\mathrm{PBE}$ by vdW correlation fully (in case of SRP32 exchange) or partly (for RPBE exchange) compensates for this. However, the inclusion of $\mathrm{vdW}$ interaction may affect other areas of the PES in different ways, and therefore the dynamics may change as well, as has been shown for $\mathrm{CHD}_{3}+\operatorname{Pt}(111)$. $^{5}$

When the ammonia molecule is fully relaxed at the surface an adsorption energy of $75.7 \mathrm{~kJ} / \mathrm{mol}$ is obtained (Figure S2 and Table S3), which is in reasonable agreement with the experiment $(88.7 \mathrm{~kJ} / \mathrm{mol}) .{ }^{50}$ Moreover, allowing the surface atoms to relax in response to the molecule as well yields an adsorption energy of $81.4 \mathrm{~kJ} / \mathrm{mol}$ (Table S3), which is in even better agreement with the experiment. When we also take into account the interaction energy of $3.0 \mathrm{~kJ} / \mathrm{mol}$ because of the employed vacuum distance (see Section 2), an adsorption energy of $84.4 \mathrm{~kJ} / \mathrm{mol}$ is obtained (Table S3), which reproduces the experiment with almost chemical accuracy. With the PBE functional a similar adsorption energy $(83.5 \mathrm{~kJ} /$ $\mathrm{mol})^{26}$ is obtained. We attribute this similarity in adsorption energy to the $\mathrm{PBE}$ exchange typically binding more than the $\mathrm{RPBE}$ exchange, combined with the vdW correlation compensating for this effect. Furthermore, the preferred adsorption site is the top site, which is in agreement with theory and experiments by Maier et al. ${ }^{51}$ and $\mathrm{Hu}$ et al., ${ }^{26}$ who both used the $\mathrm{PBE}$ xc-functional without long-range correlation effects.

We can also correct the barrier heights computed with the RPBE-vdW-DF1 functional for the unconverged value of the vacuum distance, obtaining $E_{\mathrm{b}}^{\mathrm{c}}$. Values of $E_{\mathrm{b}}^{\mathrm{c}}$ and the associated zero-point energy corrected values are listed in Tables 2 and 3.

3.2. Sticking Probability. The computed sticking probability of ammonia on $\mathrm{Ru}(0001)$ is shown in Figure 3a. The sticking probabilities computed by $\mathrm{Hu}$ et al. for vibrationally ground state $\mathrm{NH}_{3}{ }^{26}$ and the sticking probabilities measured by Mortensen et al. ${ }^{25}$ are also shown. The sticking

Table 2. Minimum Barrier Geometries and Barrier Heights of Ammonia on $\mathrm{Ru}(0001)^{a}$

\begin{tabular}{|c|c|c|c|c|c|c|c|}
\hline barrier & $Z_{\mathrm{N}}^{\ddagger}(\AA)$ & $r^{\ddagger}(\AA)$ & $\theta_{1}^{\ddagger}(\mathrm{deg})$ & $\beta^{\ddagger}(\mathrm{deg})$ & $\gamma^{\ddagger}(\mathrm{deg})$ & $E_{\mathrm{b}}(\mathrm{kJ} / \mathrm{mol})$ & $E_{\mathrm{b}}^{\mathrm{c}}(\mathrm{kJ} / \mathrm{mol})$ \\
\hline top $2 \mathrm{fcc}$ & 2.04 & 1.62 & 115.6 & 177.2 & 61.6 & $65.9(50.0)$ & $62.9(47.0)$ \\
\hline top2hcp & 2.03 & 1.60 & 116.1 & 176.5 & 60.3 & $63.2(47.4)$ & $60.2(44.4)$ \\
\hline top2fcc (SRP32-vdW) & 2.01 & 1.62 & 113.8 & 177.6 & 63.9 & $41.4(26.1)$ & $38.4(23.1)$ \\
\hline top2hcp (SRP32-vdW) & 2.01 & 1.59 & 114.8 & 175.0 & 60.2 & $42.0(26.9)$ & $39.0(23.9)$ \\
\hline top2fcc $(\mathrm{PBE})$ & 1.99 & 1.63 & 115.1 & 176.9 & 61.8 & $43.3(29.6)$ & \\
\hline top2fcc $(\mathrm{PBE})^{26}$ & 2.06 & 1.64 & & & & $45.6(31.8)$ & \\
\hline top2hcp (PBE) & 1.99 & 1.61 & 115.6 & 176.2 & 60.6 & $40.2(26.7)$ & \\
\hline
\end{tabular}

${ }^{a}$ The zero-point energy corrected barriers are given in the brackets. Barriers corrected for the usage of a too small vacuum distance are indicated by $E_{\mathrm{b}}^{\mathrm{c}}$. 
Table 3. top2fcc and top2hcp Barrier Geometries of Ammonia on Ru(0001) Using Different xc-Functionals ${ }^{a}$

\begin{tabular}{|c|c|c|c|c|c|c|}
\hline & top $2 \mathrm{fcc}$ & top $2 \mathrm{hcp}$ & top2fcc(SRP32-vdW) & top2hcp(SRP32-vdW) & top2fcc $(\mathrm{PBE})$ & top2hcp (PBE) \\
\hline$\Delta X_{\mathrm{N}}(\AA)$ & 0.47 & 0.00 & 0.51 & 0.00 & 0.45 & 0.00 \\
\hline$\Delta Y_{\mathrm{N}}(\AA)$ & -0.28 & -0.56 & -0.29 & -0.60 & -0.25 & -0.53 \\
\hline$Z_{\mathrm{N}}(\AA)$ & 2.04 & 2.03 & 2.01 & 2.01 & 1.99 & 1.99 \\
\hline$r_{\mathrm{NH} 1}(\AA)$ & 1.62 & 1.60 & 1.59 & 1.62 & 1.63 & 1.61 \\
\hline$r_{\mathrm{NH} 2}(\AA)$ & 1.02 & 1.02 & 1.02 & 1.02 & 1.02 & 1.02 \\
\hline$r_{\mathrm{NH} 3}(\AA)$ & 1.02 & 1.02 & 1.02 & 1.03 & 1.02 & 1.02 \\
\hline$\theta_{\mathrm{NH} 1}(\mathrm{deg})$ & 115.6 & 116.1 & 114.8 & 113.8 & 115.1 & 115.6 \\
\hline$\theta_{\mathrm{NH} 2}(\mathrm{deg})$ & 55.2 & 55.2 & 55.2 & 54.6 & 55.8 & 55.6 \\
\hline$\theta_{\mathrm{NH} 3}(\mathrm{deg})$ & 55.3 & 55.2 & 55.4 & 55.9 & 55.5 & 55.7 \\
\hline$\phi_{\mathrm{NH} 1}(\operatorname{deg})$ & -30.0 & -89.9 & -29.7 & -88.0 & -30.1 & -90.1 \\
\hline$\phi_{\mathrm{NH} 2}(\operatorname{deg})$ & 61.9 & 2.5 & 63.3 & 0.7 & 62.2 & 2.6 \\
\hline$\phi_{\mathrm{NH} 3}(\operatorname{deg})$ & -122.0 & 177.6 & -123.6 & 177.6 & -122.0 & 177.4 \\
\hline$E_{\mathrm{b}}(\mathrm{kJ} / \mathrm{mol})$ & $65.9(50.0)$ & $63.2(47.4)$ & $41.4(26.1)$ & $42.0(26.9)$ & $43.3(29.6)$ & $40.2(26.7)$ \\
\hline$E_{\mathrm{b}}^{\mathrm{c}}(\mathrm{kJ} / \mathrm{mol})$ & $62.9(47.0)$ & $60.2(44.4)$ & $38.4(23.1)$ & $39.0(23.9)$ & & \\
\hline
\end{tabular}

${ }^{a_{T}}$ The relative cartesian coordinates of the nitrogen atom to the closest top atom are given by $\Delta X_{\mathrm{N}}$ and $\Delta Y_{\mathrm{N}}$ and the distance between the nitrogen atom and the surface is given by $Z_{\mathrm{N}}$. The positions of the hydrogen atoms relative to the nitrogen atom are given in spherical coordinates. The zeropoint energy corrected barriers are given in the brackets and in $\mathrm{kJ} / \mathrm{mol}$. Barriers corrected for the usage of a too small vacuum distance are indicated by $E_{\mathrm{b}}^{\mathrm{c}}$.

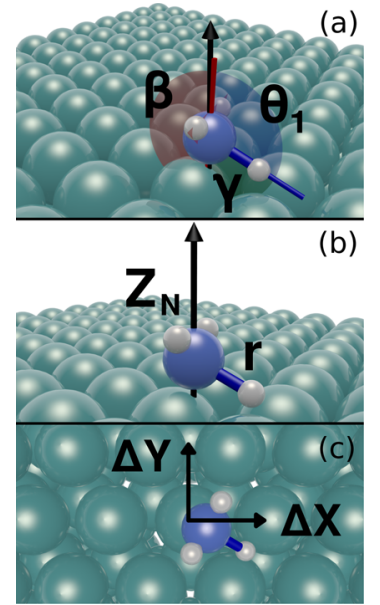

Figure 2. (a) top2fcc TS of ammonia on $\mathrm{Ru}(0001)$, indicating the orientation angles as used in Table 2. $\theta_{1}$ is the angle between the dissociating $\mathrm{NH}$ bond and the surface normal, $\beta$ is the angle between the principal axis of $\mathrm{NH}_{2}$ (i.e., the vector going from the geometric center of the two nondissociating hydrogen atoms to the nitrogen atom) and the surface normal, and $\gamma$ is the angle between $\theta_{1}$ and this principal axis. (b) Same as panel a but here the length of the dissociating $\mathrm{NH}$ bond $(r)$ and distance of the nitrogen atom to the surface $\left(Z_{\mathrm{N}}\right)$ are illustrated. (c) Top view of the top2fcc TS geometry.

probability obtained with the RPBE-vdW-DF1 functional for a mobile surface is in better agreement with the experiment than the sticking probabilities computed using the PBE functional within the static surface approximation. ${ }^{26}$ Our computed sticking probabilities are smaller than the PBE sticking probabilities even though in the calculation of the latter the contribution from excited vibrational states to the sticking was omitted, and no averaging over the velocity distribution in the molecular beam was performed. Performing both averaging procedures would have led to even higher PBE sticking probabilities (see Figure S5 for sticking probabilities for vibrationally ground state $\mathrm{NH}_{3}$ ). Furthermore, when the experimental results are multiplied with a factor 1.5 (Figure $3 \mathrm{~b}$ ), an excellent agreement between our computed and the measured sticking probabilities is obtained. This multiplication

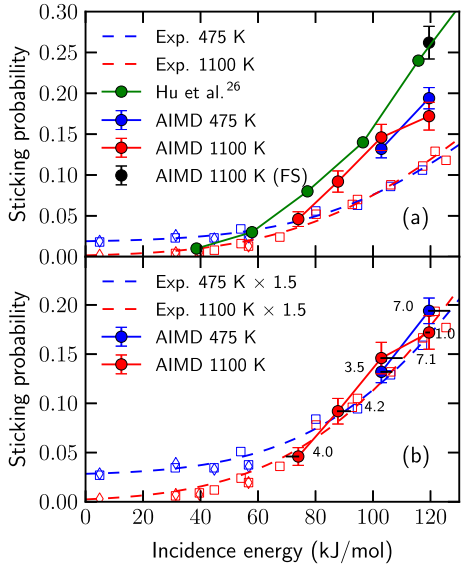

Figure 3. (a) Sticking probability of ammonia on $\mathrm{Ru}(0001)$. All the theoretical results are indicated by closed circles and the experimental results are indicated by open diamonds and squares, of which the diamonds and squares are measurements using hydrogen or nitrogen desorption, respectively. Experimental results are taken from ref 25, and previous theoretical results without surface motion (closed green circles) are from ref 26 . The AIMD results are the closed blue and red circles. $T_{\mathrm{s}}=475$ and $1100 \mathrm{~K}$ are represented by the blue and red symbols, respectively. The error bars represent $68 \%$ confidence intervals. (b) Same as panel a, but with the experimental results multiplied with a factor 1.5. The horizontal offsets between the computed and fitted experimental sticking probabilities are indicated by the numbers.

improves the mean absolute deviation (the mean of the distances between the theoretical and experimental sticking probability curves along the energy axis) from 23.1 to $4.5 \mathrm{~kJ} /$ mol, which is almost within chemical accuracy. Moreover, in agreement with the experiment, no difference in reactivity is obtained using a surface temperature of 475 or $1100 \mathrm{~K}$ at high incidence energy, that is, the sticking probability has no surface temperature dependence. Trapping is also observed (see Figure S4), but the trapped molecules will most likely desorb when the corresponding trajectories are propagated longer for the incidence energies considered, where the measured sticking is independent of the surface temperature. 
Table 4. Average Value of the $\theta_{1}, \beta$, and $\gamma$ Angles with the Standard Error $\left(\sigma_{\mathrm{m}}\right)$ and Standard Deviation $(\sigma)$ for All the Reacted and Scattered Trajectories ${ }^{a}$

$\begin{array}{llll} & \theta_{1}(\mathrm{deg}) \pm \sigma_{\mathrm{m}}(\sigma) & \beta(\mathrm{deg}) \pm \sigma_{\mathrm{m}}(\sigma) & \gamma(\mathrm{deg}) \pm \sigma_{\mathrm{m}}(\sigma) \\ \text { reacted }(t=0) & 41.0 \pm 1.1(25.5) & 62.2 \pm 1.1(26.6) & 62.3 \pm 0.4(9.8) \\ \text { reacted }\left(r=r^{\ddagger}\right) & 103.6 \pm 0.5(12.5) & 155.2 \pm 0.6(13.4) & 74.6 \pm 0.8(19.1) \\ \text { scattered }(t=0) & 89.7 \pm 0.7(38.4) & 90.6 \pm 0.7(40.1) & 62.2 \pm 0.2(9.8) \\ \text { top2fcc } & 115.6 & 177.2 & 61.6 \\ \text { top2hcp } & 116.1 & 176.5 & 60.3\end{array}$

${ }^{a_{T}}$ The top2fcc and top2hcp TS values are included as well.

The effect of surface motion is investigated as well by fixing the surface atoms in their ideal positions, commonly referred to as a frozen surface or Born-Oppenheimer static surface model, but using a lattice expansion coefficient corresponding to $T_{\mathrm{s}}=1100 \mathrm{~K}$. This excludes any energy transfer from ammonia to the surface atoms, and corrugation in barrier heights and positions because of the movement of the surface atoms. In Figure $3 a$ the sticking probability on the frozen surface is considerably higher than on the mobile surface. The thermal modulation of barrier heights and positions typically has a negligible effect on the sticking probability when the incidence energy is near or above the barrier height, as has been shown previously for methane reacting on several surfaces. ${ }^{8,52,53}$ Furthermore, since both in this work and the experiments performed by Mortensen et al. ${ }^{25}$ no surface temperature dependence is found for the incidence energies addressed, we expect that this holds true for $\mathrm{NH}_{3}+\mathrm{Ru}(0001)$ as well. We therefore suggest that the increase in sticking is mainly caused by the lack of energy transfer from the molecule to the surface atoms, and thus including surface motion into the modeling of $\mathrm{NH}_{3}+\mathrm{Ru}(0001)$ is necessary.

The difference between our computed sticking probabilities and those by $\mathrm{Hu}$ et $\mathrm{al}^{26}$ for vibrationally ground state ammonia is somewhat smaller than might have been expected from the difference between the $E_{\mathrm{b}}$ value of PBE $(45.6 \mathrm{~kJ} /$ $\mathrm{mol})^{26}$ and RPBE-vdW-DF1 $(62.9 \mathrm{~kJ} / \mathrm{mol})$. However, we note that the sticking probability of $\mathrm{Hu}$ et al. should be underestimated as also the contribution of vibrationally excited $\mathrm{NH}_{3}$ should be taken into account (see Figure S5). Furthermore, as we will show in the next section, the dynamics plays an important role for the dissociation of ammonia, especially the orientation of ammonia. Therefore, the minimum barrier height might play a smaller role than expected.

The sticking probabilities measured on $\mathrm{Ru}(0001)$ were not absolute sticking probabilities, but relative sticking probabilities measured by a combination of three different methods using partial pressures and temperature-programmed desorption of $\mathrm{H}_{2}$ and $\mathrm{N}_{2}$. The relative sensitivities of these three methods were calibrated to one another for the overlapping regimes of surface temperatures where the methods were applicable. Absolute sticking probabilities were then obtained by also performing a King and Wells experiment ${ }^{54}$ on a surface with an artificially high defect concentration created by sputtering, against which the other methods for measuring sticking of $\mathrm{NH}_{3}$ on defect-free $\mathrm{Ru}(0001)$ were then calibrated. ${ }^{25}$ This procedure was needed because of the tendency of $\mathrm{NH}_{3}$ to stick to the walls of the chamber. However, the uncertainty of the absolute sticking probabilities obtained in this manner was not stated. Because the shape of the sticking probability curve is predicted correctly if we multiply the experimental data with a factor 1.5 (see Figure $3 b$ ), and the experimental error margin is unknown, it is possible that the disagreement we note between the experiment and theory in this work is at least in part caused by an error in the calibration of the sticking probabilities. Therefore, we conclude that the computed reactivity in this work is potentially correct, but additional experiments are required in order to validate this assumption. For further discussion of the agreement between theory and experiment, see the Supporting Information.

3.3. Dynamics during the Reaction. In order to elucidate the reaction mechanism of ammonia on $\mathrm{Ru}(0001)$, we now look at the dynamics of the reaction. First, all the reacted trajectories, except for one, occurred without bouncing, so the reaction proceeds directly, without the system going through a long-lived molecular precursor state. However, looking at the angles of ammonia during a reaction (see Figure 1 and Table 4, and Figure $4 a-d$, which present snapshots from

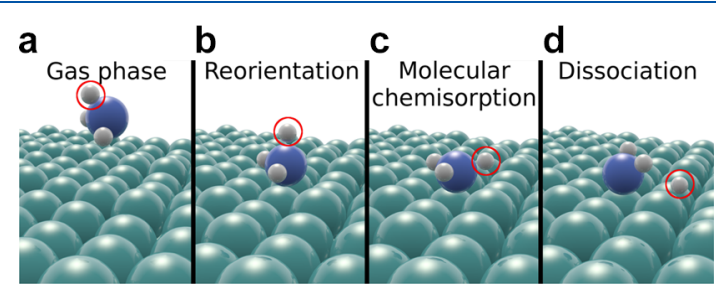

Figure 4. Snapshots from a typical trajectory of reacting ammonia on $\mathrm{Ru}(0001)$. The gas phase (a), first moment of reorientation (b), molecular chemisorbed state (c), and moment of reaction (d) are depicted. The dissociating hydrogen atom is indicated by the red circles. The simulation time portrayed in these panels is about $50 \mathrm{fs}$.

a representative example of the reactive trajectories) paints a different picture. The initial distribution of $\theta_{1}$ is centered on values smaller than $90^{\circ}$ (Figure $1 \mathrm{~b}$ ), so typically the leaving $\mathrm{H}$ atom initially points away from the surface (Figure 4a). The dissociating hydrogen atom is only reoriented toward the surface (as in the TS, see Figure 2a) near the moment of the reaction (i.e., when $r=r^{\ddagger}$ ), see Figures $1 \mathrm{~b}$ and $4 \mathrm{~d}$. Moreover, the nondissociating hydrogen atoms are initially oriented toward the surface $\left(\theta_{2,3}>90^{\circ}\right.$ and $\beta<90^{\circ}$, Figures $1 \mathrm{c}$, d and $4 \mathrm{a})$, while at the time of reaction they point upward $\left(\theta_{2,3}<90^{\circ}\right.$ and $\beta>90^{\circ}$, Figures $1 \mathrm{c}, \mathrm{d}$ and $4 \mathrm{~d}$ ). Closer inspection of the reacted trajectories suggests that the nitrogen atom first binds to the surface (Figure $4 b, c$ ), while the two nondissociating hydrogen atoms are oriented along (Figure $4 \mathrm{~b}$ ) and then away from (Figure 4c) the surface. After this, a rapid reorientation of all the hydrogen atoms occurs and subsequently a hydrogen atom dissociates (Figure 4d) and this is the hydrogen atom that was originally oriented away from the surface (Figure 4a). It is possible that by first binding the nitrogen atom to the surface, and thus forming a chemisorbed molecule, the $\mathrm{NH}$ bond is destabilized so that it can dissociate more easily after reorientation. Furthermore, this reaction occurs rapidly and 
without bounces on the surface, where typically the time between ammonia impacting on the surface and dissociation of a $\mathrm{NH}$ bond is about 50-150 fs. Several movies of dissociation events are provided in the Supporting Information to illustrate the mechanism. Since the dissociative chemisorption of ammonia on $\mathrm{Ru}(0001)$ occurs initially through molecular chemisorption and only subsequently a $\mathrm{NH}$ bond dissociates, we argue that this is not a simple direct reaction mechanism as reported before, ${ }^{25,26}$ but rather a molecular chemisorptionmediated direct reaction mechanism.

Importantly, at the initial time step the orientation distribution of the reacting molecule is nonstatistical, that is, it does not resemble a $\sin (\theta)$ distribution as shown in Figure 1a. A nonstatistical initial distribution has also been observed for other polyatomic molecules reacting on metal surfaces (Figure 1a), for example, $\mathrm{CHD}_{3}{ }^{15,17,49}$ and methanol, ${ }^{7}$ for which cases the reaction mechanism can be described reasonably well as a rotational sudden mechanism. Interestingly, unlike for the aforementioned cases, the initial orientation distribution of $\mathrm{NH}_{3}$ does not resemble the barrier geometry, or the orientation distribution at the time of reaction. Only at the moment of dissociation, that is, when $r=$ $r^{\ddagger}$ for the dissociating bond, does the time-evolved orientation distribution of ammonia resemble the barrier geometry, which to the best of our knowledge has not been observed before. This has consequences for the approximations that can be made in modeling the reaction. For example, the HOD + $\mathrm{Ni}(111)$ reaction can be treated as rotationally adiabatic, ${ }^{4,55}$ while the reaction of $\mathrm{CHD}_{3}+\mathrm{Ni}(111)$ can be treated reasonably well with a rotational sudden approximation ${ }^{14}$ (see also Figure 1a). However, the unique behavior of ammonia, where rotationally nonadiabatic dynamics is coupled with a nonstatistical distribution of the orientation of the reacting molecule at $t=0$, prevents the usage of such approximations and only models where the full dynamics is included, such as AIMD, can describe $\mathrm{NH}_{3}+\mathrm{Ru}(0001)$ correctly. Furthermore, quantum dynamics (QD) might be necessary to describe the reaction of $\mathrm{NH}_{3}$ on $\mathrm{Ru}(0001)^{26}$ at low $E_{\mathrm{j}}$, but performing $\mathrm{QD}$ calculations using the full $12 \mathrm{D}$ hamiltonian is probably computationally prohibitive. So far $\mathrm{QD}$ employing a hamiltonian including all degrees of freedom of the molecule has been performed up to 9D, for example, on $\mathrm{H}_{2} \mathrm{O}+$ $\mathrm{Cu}(111) .{ }^{56}$ For molecules with more than nine degrees of freedom, reduced dimensionality hamiltonians have been employed, for example, $\mathrm{CH}_{4}+\mathrm{Ni}(111),{ }^{57}$ and the use of the rotationally adiabatic or rotational sudden approximation might therefore be desirable. However, as we have noted, these approximations are not valid for the reaction of $\mathrm{NH}_{3}$ on $\mathrm{Ru}(0001)$, and therefore employing a reduced dimensionality hamiltonian may not be straightforward.

Figure 5 shows the predicted energy transfer of the scattered trajectories obtained with AIMD and by the refined Baule model, ${ }^{58,59}$ which is defined as

$$
\left\langle E_{\mathrm{T}}\right\rangle=\frac{2.4 \mu}{(1+\mu)^{2}}\left\langle E_{\mathrm{i}}\right\rangle
$$

where $\mu=m / M$ ( $m$ is the mass of ammonia and $M$ is the mass of a ruthenium atom). Here we see that the energy transfer computed with AIMD is about $20 \%$, whereas the refined Baule model predicts an energy transfer of about $30 \%$. This disagreement is larger than what has generally been observed for $\mathrm{CHD}_{3}{ }^{59}$ and methanol. ${ }^{7}$ It is possible that trajectories that transfer less energy from ammonia to the metal surface are also

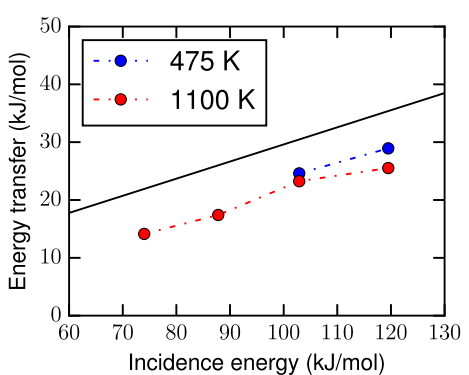

Figure 5. Average energy transfer from ammonia to $\mathrm{Ru}(0001)$ compared to the refined Baule model. The black line is the energy transfer predicted by the refined Baule model, whereas the blue and red circles indicate the energy transfer predicted by AIMD at $T_{\mathrm{s}}=475$ and $1100 \mathrm{~K}$, respectively.

less likely to trap, and therefore the average energy transfer is lower than one would expect from the comparatively simple refined Baule model.

Molecules are most likely to trap when the lone pair on the nitrogen atom is pointing away from the surface $\left(\beta^{\prime}<90^{\circ}\right.$, see Figure 1e). When the lone pair is oriented more toward the surface, it is considerably more likely to react, probably because of the possibility of forming the chemisorption-like state required for the reaction. An obvious reason for trapping would be the translational energy transfer from ammonia to the metal surface. However, even when energy transfer from the molecule to the surface is not allowed by employing a frozen surface, trapping is still observed (about $2 \%$ at $\left\langle E_{\mathrm{i}}\right\rangle=$ $119 \mathrm{~kJ} / \mathrm{mol}$, versus $5 \%$ if surface motion is included, see Figure S4). This suggests that energy transfer to other motions, that is, motions of $\mathrm{NH}_{3}$, may also cause ammonia to be trapped. For instance, it is possible to excite the vibrational bending mode of ammonia when the umbrella is pointing toward the surface, thereby converting translational energy into vibrational energy. Also, a large fraction of the translational energy of trapped molecules is oriented parallel to the surface instead of perpendicular to the surface after the initial collision. These energy transfer effects are quantified in Figure 6. The largest energy transfer is observed to vibrations and rotations, and this represents about $60 \%$ of the energy transferred. The energy transfer from motion normal to the surface to motion parallel to the surface and to the phonons and rovibrational motion

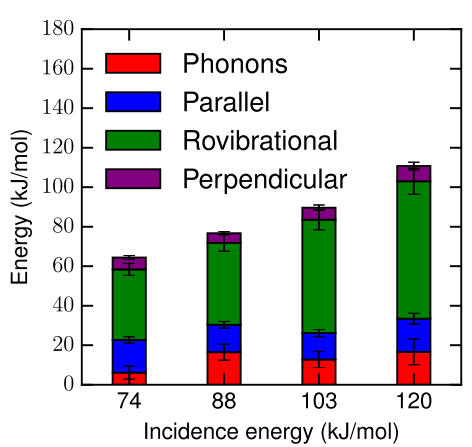

Figure 6. Kinetic energy of ammonia parallel ( $X Y$ direction, blue) and perpendicular ( $Z$ direction, purple) to the surface, the energy transferred from $\mathrm{NH}_{3}$ to the surface phonons (compared to $t=0$, red), and increase in the rovibrational energy of $\mathrm{NH}_{3}$ (compared to $t$ $=0$, green) at the final time step, that is, when $t=1.0 \mathrm{ps}$, as a function of incidence energy. The error bars represent $68 \%$ confidence intervals. 
make it less likely to scatter. It is expected that these trapped trajectories are likely to scatter back into the gas phase at longer simulation times, as they might be able at some point to escape both the chemisorption and physisorption wells. This has also been observed for some of the scattered trajectories, which scattered after one or two bounces on the surface. The observation of trajectories that scattered after one or two bounces, and the observation that only one of the reacted trajectories was indirect (occurred with bouncing) is in accordance with the original proposal by the experimentalists of a direct mechanism for the high $E_{\mathrm{i}}$ addressed here, and the surface temperature independence of reaction they observed.

Figure 7 shows that the reaction occurs relatively more often near (i.e., more than expected on the basis of relative surface

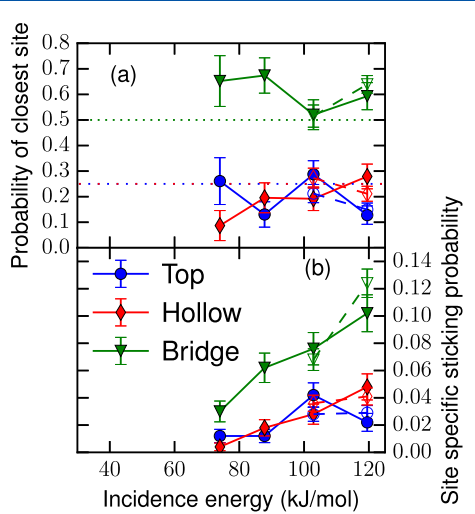

Figure 7. (a) Fraction of the closest high symmetry site, that is, the top, hollow, and bridge (blue, red, and green, respectively) sites, to the impact site of reacting ammonia as a function of the incidence energy when a bond dissociates, that is, when $r=r^{\ddagger}$. The open and closed symbols indicate a surface temperature of 475 and $1100 \mathrm{~K}$, respectively. The dotted lines indicate the statistical average for the high symmetry sites. (b) Sticking probability of $\mathrm{NH}_{3}$ on the high symmetry sites as a function of the incidence energy. The error bars represent $68 \%$ confidence intervals.

areas) the bridge site than near the top and hollow sites. However, from the minimum barrier location (near the top site, see Section 3.1) one would expect that the top site should be relatively more reactive than the other sites. The barrier height on the bridge site was predicted by $\mathrm{Hu}$ et al. to be 11 $\mathrm{kJ} / \mathrm{mol}$ higher than the minimum barrier height. ${ }^{26}$ Furthermore, the reactivity of the top and hollow sites is similar, even though compared to the minimum barrier, the barriers on the hcp and fcc sites were predicted to be higher by 49 and $38 \mathrm{~kJ} /$ mol, respectively. ${ }^{26}$ We have considered the possibility that the relatively low reactivity near the top site is caused by the bobsled effect, ${ }^{60,61}$ which can reduce the reactivity as it causes the molecule to slide off the MEP and react over a higher barrier than the lowest barrier. ${ }^{8,59}$ However, Figure 8 shows that the average distance of the nitrogen atom to the surface is close to the TS value for all the three sites. Therefore, it seems unlikely that the bobsled effect plays a large role. At present, it remains unclear why the bridge site is more reactive than the top and hollow sites. We speculate that the use of vdW-DF1 correlation leads to the barriers being relatively lower (i.e., compared to PBE) above the bridge and hollow sites, where the barriers are closer to the surface.

Interestingly, a considerable amount of steering in the $\mathrm{XY}$ plane is observed (see Figure 9). The steering also seems to be independent of incidence energy and whether the trajectory

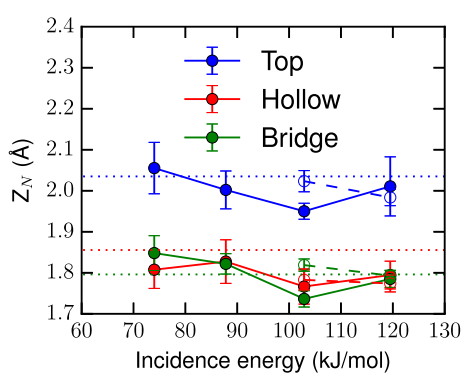

Figure 8. Distance of the nitrogen atom to the surface when a bond dissociates, that is, when $r=r^{\ddagger}$, as a function of incidence energy. The open and closed circles indicate a surface temperature of 475 and $1100 \mathrm{~K}$, respectively. The blue, red, and green lines indicate the top, hollow, and bridge sites. The horizontal dotted lines indicate the TS values of $Z_{\mathrm{N}}$, where the values belonging to the hollow and bridge sites are taken from ref 26 . The error bars represent $68 \%$ confidence intervals.

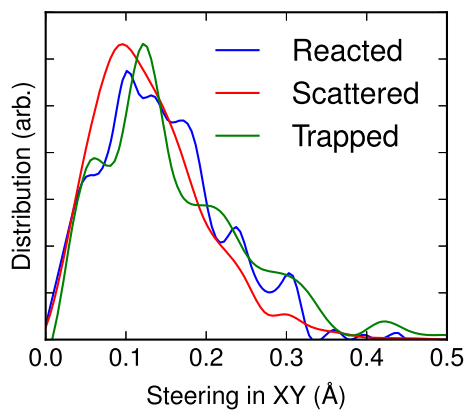

Figure 9. Distribution of steering of ammonia in the $X Y$ direction for reacted (blue), scattered (red), and trapped (green) trajectories. Steering is here defined as the distance travelled by $\mathrm{NH}_{3}$ in the $X Y$ plane between the initial time step and first classical turning point for the scattered and trapped trajectories, and between the initial time step and when a bond dissociates, i.e., when $r=r^{\ddagger}$, for reacted trajectories.

will go on to react, scatter, or trap. Moreover, looking at Figure 10 , the dynamical steering in the $X Y$ direction mostly steers ammonia away from the hollow sites toward the bridge sites. At high incidence energy steering from the bridge site toward the top site occurs as well.

\section{CONCLUSIONS}

To summarize, in this work the dissociative chemisorption of ammonia on $\mathrm{Ru}(0001)$ is investigated with AIMD. Not only is surface motion included for this reaction for the first time, but also a functional incorporating attractive van der Waals correlation (RPBE-vdW-DF1) is employed as well. With respect to the earlier work employing the PBE functional and modeling the $\mathrm{Ru}(0001)$ surface as static, the computed sticking probability is found to be in improved agreement with the experiment. This improvement is attributed to both modeling the $\mathrm{Ru}(0001)$ as a mobile surface, and using the RPBE-vdWDF1 functional. Also, the lack of the surface temperature dependence at high incidence energy observed by the experiment is confirmed with AIMD. We find that the modeling of surface motion is required to accurately describe the sticking probability. The reaction mechanism is neither rotationally adiabatic nor rotational sudden, with initial and intermediate (i.e., at time of reaction) orientation distributions that are both nonstatistical, but do not resemble one another, 


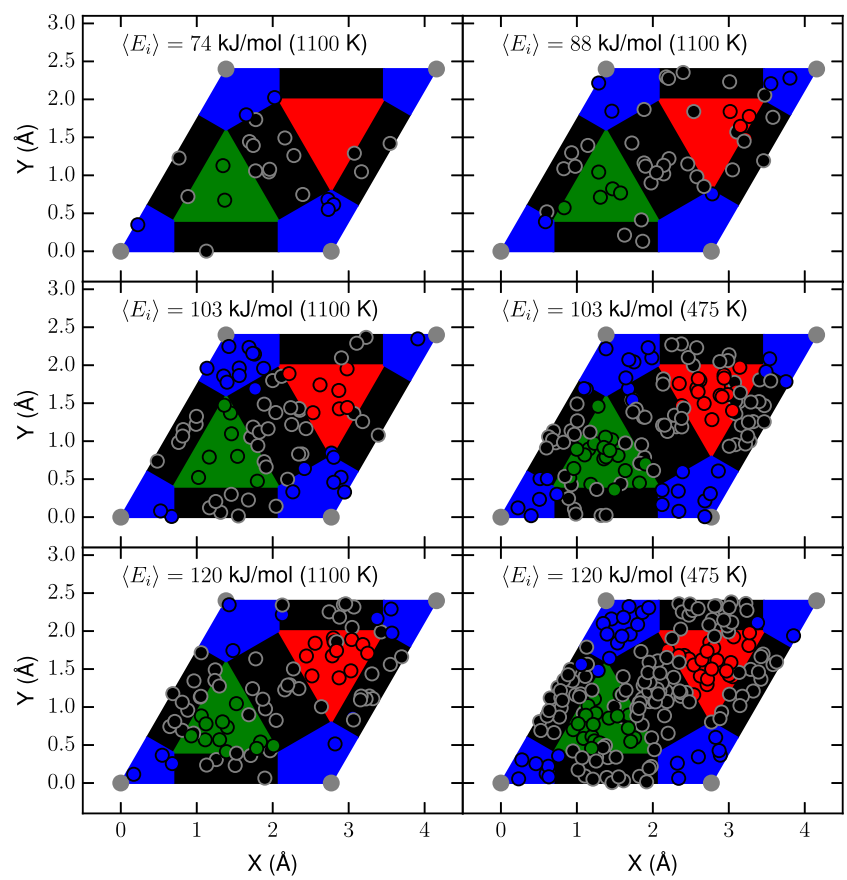

Figure 10. Impact site of reacting ammonia on $\mathrm{Ru}(0001)$ indicated with circles at the initial time step, that is, $t=0$ fs and $Z_{\mathrm{COM}}=7.5 \AA$, whereas the color of the circle indicates the closest high symmetry site when a bond dissociates, i.e., when $r=r^{\ddagger}$. The top, fcc, hcp, and bridge sites are indicated in blue, green, red, and black, respectively, and the top layer atoms are indicated by the gray circles.

which to the best of our knowledge has not been observed before. Furthermore, it is observed that under the conditions investigated the dissociation of ammonia on $\mathrm{Ru}(0001)$ is not described by a simple direct, or by an indirect trappingmediated reaction mechanism, but rather by a direct reaction mechanism in which $\mathrm{NH}_{3}$ goes through a very short-lived molecularly chemisorbed state. Direct dissociative chemisorption of a polyatomic molecule where the molecular chemisorption of a molecule is immediately followed by dissociation has also not been observed before.

\section{ASSOCIATED CONTENT}

\section{S Supporting Information}

The Supporting Information is available free of charge on the ACS Publications website at DOI: 10.1021/acs.jpcc.9b09121.

Convergence of the minimum barrier; molecular chemisorption and physisorption wells of $\mathrm{NH}_{3}$; elbow plot of the PES; trapping probabilities; reaction probability of vibrational ground state ammonia; adsorption energy of $\mathrm{NH}_{3}$; and beam parameters describing the simulated $\mathrm{NH}_{3}$ velocity distributions (PDF)

\section{AUTHOR INFORMATION}

\section{Corresponding Authors}

*E-mail: n.gerrits@lic.leidenuniv.nl (N.G.).

*E-mail: g.j.kroes@chem.leidenuniv.nl (G.-J.K.).

ORCID

Nick Gerrits: 0000-0001-5405-7860

Geert-Jan Kroes: 0000-0002-4913-4689

Notes

The authors declare no competing financial interest.

\section{ACKNOWLEDGMENTS}

This work has been financially supported through an NWO/ CW TOP Grant (no. 715.017.001). Furthermore, this work was carried out on the Dutch national supercomputer with the support of NWO-EW. The authors thank Prof. Alan Luntz and Dr. Helen Chadwick for useful discussions.

\section{REFERENCES}

(1) Lin, X.; Yoon, Y.; Petrik, N. G.; Li, Z.; Wang, Z.-T.; Glezakou, V.-A.; Kay, B. D.; Lyubinetsky, I.; Kimmel, G. A.; Rousseau, R.; et al. Structure and Dynamics of $\mathrm{CO}_{2}$ on Rutile $\mathrm{TiO}_{2}(110)-1 \times 1$. J. Phys. Chem. C 2012, 116, 26322-26334.

(2) Naderian, M.; Groß, A. From Single Molecules to Water Networks: Dynamics of Water Adsorption on Pt(111). J. Chem. Phys. 2016, 145, 094703.

(3) Zhou, X.; Kolb, B.; Luo, X.; Guo, H.; Jiang, B. Ab Initio Molecular Dynamics Study of Dissociative Chemisorption and Scattering of $\mathrm{CO}_{2}$ on $\mathrm{Ni}(100)$ : Reactivity, Energy Transfer, Steering Dynamics, and Lattice Effects. J. Phys. Chem. C 2017, 121, 55945602.

(4) Migliorini, D.; Nattino, F.; Tiwari, A. K.; Kroes, G.-J. HOD on $\mathrm{Ni}(111)$ : Ab Initio Molecular Dynamics Prediction of Molecular Beam Experiments. J. Chem. Phys. 2018, 149, 244706.

(5) Chadwick, H.; Migliorini, D.; Kroes, G. J. $\mathrm{CHD}_{3}$ Dissociation on $\mathrm{Pt}(111)$ : A Comparison of the Reaction Dynamics Based on the PBE Functional and on a Specific Reaction Parameter Functional. J. Chem. Phys. 2018, 149, 044701.

(6) Migliorini, D.; Chadwick, H.; Kroes, G.-J. Methane on a Stepped Surface: Dynamical Insights on the Dissociation of $\mathrm{CHD}_{3}$ on $\mathrm{Pt}(111)$ and $\mathrm{Pt}(211)$. J. Chem. Phys. 2018, 149, 094701.

(7) Gerrits, N.; Kroes, G.-J. An AIMD Study of Dissociative Chemisorption of Methanol on $\mathrm{Cu}(111)$ with Implications for Formaldehyde Formation. J. Chem. Phys. 2019, 150, 024706.

(8) Gerrits, N.; Shakouri, K.; Behler, J.; Kroes, G.-J. Accurate Probabilities for Highly Activated Reaction of Polyatomic Molecules on Surfaces Using a High-Dimensional Neural Network Potential: $\mathrm{CHD}_{3}+\mathrm{Cu}(111)$. J. Phys. Chem. Lett. 2019, 10, 1763-1768.

(9) Zhang, Y.; Zhou, X.; Jiang, B. Bridging the Gap between Direct Dynamics and Globally Accurate Reactive Potential Energy Surfaces Using Neural Networks. J. Phys. Chem. Lett. 2019, 10, 1185-1191.

(10) Farjamnia, A.; Jackson, B. The Dissociative Chemisorption of Water on Ni(111): Mode- and Bond-Selective Chemistry on Metal Surfaces. J. Chem. Phys. 2015, 142, 234705.

(11) Jiang, B. Rotational and Steric Effects in Water Dissociative Chemisorption on $\mathrm{Ni}(111)$. Chem. Sci. 2017, 8, 6662-6669.

(12) Campbell, V. L.; Chen, N.; Guo, H.; Jackson, B.; Utz, A. L. Substrate Vibrations as Promoters of Chemical Reactivity on Metal Surfaces. J. Phys. Chem. A 2015, 119, 12434-12441.

(13) Guo, H.; Farjamnia, A.; Jackson, B. Effects of Lattice Motion on Dissociative Chemisorption: Toward a Rigorous Comparison of Theory with Molecular Beam Experiments. J. Phys. Chem. Lett. 2016, 7, 4576-4584.

(14) Jackson, B.; Nattino, F.; Kroes, G.-J. Dissociative Chemisorption of Methane on Metal Surfaces: Tests of Dynamical Assumptions Using Quantum Models and Ab Initio Molecular Dynamics. J. Chem. Phys. 2014, 141, 054102. 
(15) Gerrits, N.; Migliorini, D.; Kroes, G.-J. Dissociation of $\mathrm{CHD}_{3}$ on $\mathrm{Cu}(111), \mathrm{Cu}(211)$, and Single Atom Alloys of $\mathrm{Cu}(111)$. J. Chem. Phys. 2018, 149, 224701.

(16) Jackson, B.; Nave, S. The Dissociative Chemisorption of Methane on $\mathrm{Ni}(100)$ : Reaction Path Description of Mode-Selective Chemistry. J. Chem. Phys. 2011, 135, 114701.

(17) Nattino, F.; Migliorini, D.; Kroes, G.-J.; Dombrowski, E.; High, E. A.; Killelea, D. R.; Utz, A. L. Chemically Accurate Simulation of a Polyatomic Molecule-Metal Surface Reaction. J. Phys. Chem. Lett. 2016, 7, 2402-2406.

(18) Ertl, G. Primary Steps in Catalytic Synthesis of Ammonia. J. Vac. Sci. Technol., A 1983, 1, 1247-1253.

(19) Nielsen, A. Ammonia: Catalysis and Manufacture; Springer Science \& Business Media, 2012.

(20) Erisman, J. W.; Sutton, M. A.; Galloway, J.; Klimont, Z.; Winiwarter, W. How a Century of Ammonia Synthesis Changed the World. Nat. Geosci. 2008, 1, 636-639.

(21) Choudhary, T. V.; Sivadinarayana, C.; Goodman, D. W. Catalytic Ammonia Decomposition: COx-Free Hydrogen Production for Fuel Cell Applications. Catal. Lett. 2001, 72, 197-201.

(22) Schüth, F.; Palkovits, R.; Schlögl, R.; Su, D. S. Ammonia as a Possible Element in an Energy Infrastructure: Catalysts for Ammonia Decomposition. Energy Environ. Sci. 2012, 5, 6278-6289.

(23) Mukherjee, S.; Devaguptapu, S. V.; Sviripa, A.; Lund, C. R. F.; $\mathrm{Wu}, \mathrm{G}$. Low-Temperature Ammonia Decomposition Catalysts for Hydrogen Generation. Appl. Catal., B 2018, 226, 162-181.

(24) Bradford, M. C. J.; Fanning, P. E.; Vannice, M. A. Kinetics of NH Decomposition over Well Dispersed Ru. J. Catal. 1997, 172, 479-484.

(25) Mortensen, H.; Diekhöner, L.; Baurichter, A.; Jensen, E.; Luntz, A. C. Dynamics of Ammonia Decomposition on $\mathrm{Ru}(0001)$. J. Chem. Phys. 2000, 113, 6882-6887.

(26) Hu, X.; Yang, M.; Xie, D.; Guo, H. Vibrational Enhancement in the Dynamics of Ammonia Dissociative Chemisorption on $\mathrm{Ru}(0001)$. J. Chem. Phys. 2018, 149, 044703.

(27) Jiang, B.; Guo, H. Permutation Invariant Polynomial Neural Network Approach to Fitting Potential Energy Surfaces. III. Molecule-Surface Interactions. J. Chem. Phys. 2014, 141, 034109.

(28) Perdew, J. P.; Burke, K.; Ernzerhof, M. Generalized Gradient Approximation Made Simple. Phys. Rev. Lett. 1996, 77, 3865.

(29) Perdew, J. P.; Wang, Y. Accurate and Simple Analytic Representation of the Electron-Gas Correlation Energy. Phys. Rev. B 1992, 45, 13244-13249.

(30) Diaz, C.; Pijper, E.; Olsen, R. A.; Busnengo, H. F.; Auerbach, D. J.; Kroes, G. J. Chemically Accurate Simulation of a Prototypical Surface Reaction: $\mathrm{H}_{2}$ Dissociation on $\mathrm{Cu}(111)$. Science 2009, 326, 832-834.

(31) Wijzenbroek, M.; Kroes, G. J. The Effect of the ExchangeCorrelation Functional on $\mathrm{H}_{2}$ Dissociation on $\mathrm{Ru}(0001)$. J. Chem. Phys. 2014, 140, 084702.

(32) Nattino, F.; Ueta, H.; Chadwick, H.; van Reijzen, M. E.; Beck, R. D.; Jackson, B.; van Hemert, M. C.; Kroes, G.-J. Ab Initio Molecular Dynamics Calculations versus Quantum-State-Resolved Experiments on $\mathrm{CHD}_{3}+\mathrm{Pt}(111)$ : New Insights into a Prototypical Gas-Surface Reaction. J. Phys. Chem. Lett. 2014, 5, 1294-1299.

(33) Nattino, F.; Migliorini, D.; Bonfanti, M.; Kroes, G.-J. Methane Dissociation on $\mathrm{Pt}(111)$ : Searching for a Specific Reaction Parameter Density Functional. J. Chem. Phys. 2016, 144, 044702.

(34) Hammer, B.; Hansen, L. B.; Nørskov, J. K. Improved Adsorption Energetics within Density-Functional Theory Using Revised Perdew-Burke-Ernzerhof Functionals. Phys. Rev. B 1999, 59, 7413-7421.

(35) Dion, M.; Rydberg, H.; Schröder, E.; Langreth, D. C.; Lundqvist, B. I. Van Der Waals Density Functional for General Geometries. Phys. Rev. Lett. 2004, 92, 246401.

(36) Kresse, G.; Hafner, J. Ab Initio Molecular-Dynamics Simulation of the Liquid-Metal-Amorphous-Semiconductor Transition in Germanium. Phys. Rev. B 1994, 49, 14251-14269.
(37) Kresse, G.; Hafner, J. Ab Initio Molecular Dynamics for Liquid Metals. Phys. Rev. B 1993, 47, 558-561.

(38) Kresse, G.; Furthmüller, J. Efficient Iterative Schemes for Ab Initio Total-Energy Calculations Using a Plane-Wave Basis Set. Phys. Rev. B: Condens. Matter Mater. Phys. 1996, 54, 11169-11186.

(39) Kresse, G.; Furthmüller, J. Efficiency of Ab-Initio Total Energy Calculations for Metals and Semiconductors Using a Plane-Wave Basis Set. Comput. Mater. Sci. 1996, 6, 15-50.

(40) Kresse, G.; Joubert, D. From Ultrasoft Pseudopotentials to the Projector Augmented-Wave Method. Phys. Rev. B 1999, 59, 17581775.

(41) Blöchl, P. E. Projector Augmented-Wave Method. Phys. Rev. B 1994, 50, 17953-17979.

(42) Methfessel, M.; Paxton, A. T. High-Precision Sampling for Brillouin-Zone Integration in Metals. Phys. Rev. B 1989, 40, 36163621.

(43) Henkelman, G.; Jónsson, H. A Dimer Method for Finding Saddle Points on High Dimensional Potential Surfaces Using Only First Derivatives. J. Chem. Phys. 1999, 111, 7010-7022.

(44) Heyden, A.; Bell, A. T.; Keil, F. J. Efficient Methods for Finding Transition States in Chemical Reactions: Comparison of Improved Dimer Method and Partitioned Rational Function Optimization Method. J. Chem. Phys. 2005, 123, 224101.

(45) Kästner, J.; Sherwood, P. Superlinearly Converging Dimer Method for Transition State Search. J. Chem. Phys. 2008, 128, 014106.

(46) Xiao, P.; Sheppard, D.; Rogal, J.; Henkelman, G. Solid-State Dimer Method for Calculating Solid-Solid Phase Transitions. J. Chem. Phys. 2014, 140, 174104.

(47) Arblaster, J. W. Crystallographic Properties of Ruthenium. Platin. Met. Rev. 2013, 57, 127-136.

(48) Mondal, A.; Wijzenbroek, M.; Bonfanti, M.; Díaz, C.; Kroes, G.-J. Thermal Lattice Expansion Effect on Reactive Scattering of $\mathrm{H}_{2}$ from $\mathrm{Cu}(111)$ at $\mathrm{T}_{\mathrm{s}}=925 \mathrm{~K}$. J. Phys. Chem. A 2013, 117, 8770-8781.

(49) Migliorini, D.; Chadwick, H.; Nattino, F.; Gutiérrez-González, A.; Dombrowski, E.; High, E. A.; Guo, H.; Utz, A. L.; Jackson, B.; Beck, R. D.; et al. Surface Reaction Barriometry: Methane Dissociation on Flat and Stepped Transition-Metal Surfaces. J. Phys. Chem. Lett. 2017, 8, 4177-4182.

(50) Benndorf, C.; Madey, T. E. Adsorption and Orientation of $\mathrm{NH}_{3}$ on $\mathrm{Ru}(001)$. Surf. Sci. 1983, 135, 164-183.

(51) Maier, S.; Stass, I.; Cerda, J. I.; Salmeron, M. Bonding of Ammonia and Its Dehydrogenated Fragments on $\mathrm{Ru}(0001)$. J. Phys. Chem. C 2012, 116, 25395-25400.

(52) Luntz, A. C.; Bethune, D. S. Activation of Methane Dissociation on a $\mathrm{Pt}(111)$ Surface. J. Chem. Phys. 1989, 90, 1274-1280.

(53) Tiwari, A. K.; Nave, S.; Jackson, B. The Temperature Dependence of Methane Dissociation on $\mathrm{Ni}(111)$ and $\mathrm{Pt}(111)$ : Mixed Quantum-Classical Studies of the Lattice Response. J. Chem. Phys. 2010, 132, 134702.

(54) King, D. A.; Wells, M. G. Reaction Mechanism in Chemisorption Kinetics: Nitrogen on the $\{100\}$ Plane of Tungsten. Proc. R. Soc. Lond. A 1974, 339, 245-269.

(55) Jiang, B.; Guo, H. Dynamics of Water Dissociative Chemisorption on $\mathrm{Ni}(111)$ : Effects of Impact Sites and Incident Angles. Phys. Rev. Lett. 2015, 114, 166101.

(56) Zhang, Z.; Liu, T.; Fu, B.; Yang, X.; Zhang, D. H. FirstPrinciples Quantum Dynamical Theory for the Dissociative Chemisorption of $\mathrm{H}_{2} \mathrm{O}$ on Rigid $\mathrm{Cu}(111)$. Nat. Commun. 2016, 7, 11953.

(57) Shen, X.; Zhang, Z.; Zhang, D. H. Communication: Methane Dissociation on $\mathrm{Ni}(111)$ Surface: Importance of Azimuth and Surface Impact Site. J. Chem. Phys. 2016, 144, 101101.

(58) Goodman, F. O.; Wachman, H. Y. Formula for Thermal Accommodation Coefficient, 1966.

(59) Gerrits, N.; Chadwick, H.; Kroes, G.-J. A Dynamical Study of the Dissociative Chemisorption of $\mathrm{CHD}_{3}$ on $\mathrm{Pd}(111)$. J. Phys. Chem. C 2019, 123, 24013. 
(60) Marcus, R. A. On the Analytical Mechanics of Chemical Reactions. Quantum Mechanics of Linear Collisions. J. Chem. Phys. 1966, 45, 4493-4499.

(61) McCullough, E. A.; Wyatt, R. E. Quantum Dynamics of the Collinear $\left(\mathrm{H}_{1} \mathrm{H}_{2}\right)$ Reaction. J. Chem. Phys. 1969, 51, 1253-1254. 